\title{
Alteration of the microRNA expression profile in human osteosarcoma cells transfected with APE1 siRNA
}

\author{
N. DAI ${ }^{1}$, Z. Y. ZHONG ${ }^{1}$, Y. P. CUN ${ }^{1}$, Y. QING ${ }^{1}, C H . C H E N^{1}$, P. JIANG ${ }^{1}$, M. X. $\mathrm{LI}^{1, *}$, D. WANG ${ }^{1, *}$ \\ ${ }^{1}$ Cancer Center, Daping Hospital and Research Institute of Surgery, Third Military Medical University, Chongqing, 400042, P.R. China \\ *Correspondence: dongwang64@hotmail.com,lee800265@hotmail.com
}

Received November 5, 2012 / Accepted January 10, 2013

\begin{abstract}
Apurinic/apyrimidinic endonuclease1 (APE1), which has the dual functions of DNA repair and redox regulation, is considered to be a promising potential target in cancer treatment. Microarray and qRT-PCR were used to confirm the change of miRNA followed by analysis with comprehensive bioinformatics-based analysis. Both microarray and qRT-PCR demonstrated that 13 microRNAs (miRNAs) were significantly changed (>2-fold) in APE1 knockdown HOS cells; seven of them (hsa-miR-451, hsa-miR-1290, hsa-miR-765, hsa-miR-483-5p, hsa-miR-513a-5p, hsa-miR-129-5p and hsa-miR-31) were up-regulated and the other six (hsa-miR-29b, hsa-miR-197, has-let-7b, hsa-miR-324-5p, hsa-let-7i and hsa-miR-484) were down-regulated. Furthermore, pathway analysis showed that these miRNAs and their target genes affected by the expression of APE1 were involved in pathways relating to developmental processes, regulation of cellular processes, cell signaling (such as TGF- $\beta$, Wnt, MAPK and the p53 signaling pathway) and cancers. There are putative binding sites of NF- $\kappa B$, p53, HIF- $1 \alpha$, AP-1, PEBP2, ATF, NF-Y, Pax-2,CREB and c-Myb in the promoters of several down regulated miRNAs, indicating that APE1 may regulate miRNAs via transcription factors. Our data suggest that our understanding of the biological functions of APE1 will inevitably expand due to the novel pathways that APE1 uses to regulate gene expression through miRNAs.
\end{abstract}

Key words: APE1, microRNA, bioinformatics, microarray, osteosarcoma

Osteosarcoma is the most common primary malignant bone tumor in children and adolescents and leads to the lowest survival probabilities in pediatric cancer patients [1]. Although survival rates of osteosarcoma have improved dramatically through the application of neoadjuvant chemotherapy followed by surgical resection during the late 20th century and by approximately $68 \%$ in recent years [2], the resistance to chemotherapy agents remains an important element interfering with therapeutic effect and prognosis $[3,4]$. Current standard chemotherapy of osteosarcoma is a combination of high dose methotrexate with cisplatin, doxorubicine and ifosfamide. Most of these agents are DNA damaging agents and therefore the DNA repair pathway acts as an essential mechanism for intrinsic drug resistance.

As a key gene in the base excise repair (BER) pathway, the multifunctional, apurinic/apyrimidinic endonuclease (APE1) plays a pivotal role in DNA repair and influences the sensitivity of tumor cells to chemotherapeutic agents [5]. It is not only responsible for the repair of DNA AP sites caused by oxidative and alkylation damage, but also functions as a reduction-oxidation (redox) factor maintaining the DNA binding activity of transcription factors such as $\mathrm{p} 53, \mathrm{NF}-\kappa \mathrm{B}$, HIF-1, Myb, PAX, Egr-1 and AP-1 [6]. Previous studies have shown that aberrantly high expression of APE1, which is commonly found in a number of cancers including osteosarcoma, ovarian, cervical, prostate, lung cancer, rhabdomyosarcomas, and germ cell tumors, has been closely linked to poor prognosis and poor survival as well as resistance to DNA damaging agents like cisplatin and methyl methanesulfonate $[6,7,8]$. Knocking down APE1 expression in human osteosarcoma and colorectal cells by APE1 siRNA significantly enhanced cell sensitivity to DNA damaging agents and radiation $[8,9,10]$. However, although the essential function of APE1 has been confirmed, knowledge of its regulation or interactome is still scanty. To date, we have found several different regulatory mechanisms of the APE1 interactome including acetylation, phosphorylation, ubiquitination, and redox [11]. But all of these results do not yet fully explain the mechanism of how APE1 regulates other genes in apoptosis, angiogenesis and other various biological processes. 
Ever since microRNAs (miRNAs) entered the gene network world, it provided a new basis for interpreting the interaction and regulation of genes. miRNAs are a class of endogenous single-stranded non-coding RNAs composed of 18-25 nucleotides [12]. Through suppressing gene expression by binding to the 3'-untranslated region (3'-UTR) of target genes, they perform a large range of biological functions in a variety of organisms and are involved in development, proliferation, differentiation, apoptosis and human diseases such as cancer $[13,14]$. The potential influence of miRNAs may be involved in almost every genetic pathway. Recent studies have shown that miRNAs can interact with important cancer-related genes. For example, hsamiR-125a/b, hsa-miR-25 and hsa-miR-30d are known to target p53 and play an important role in the regulation of oncogenes [15-17]. Moreover, Lin et al reported that the hsa-miR-34s family are direct transcriptional targets of $\mathrm{p} 53$, reinforcing the growing awareness which places miRNAs in a central role in a well-known tumor-suppressor network [18]. However, until today, little reports concerning the interaction between APE1 and miRNAs are available in the current scientific literature. Recently, Kim et al reported that APE1 was able to directly cleave hsa-miR-21 and hsa-miR-10b in vitro, suggesting a novel connection between APE1 and miRNA [19]. We propose that APE1 is able to indirectly mediate miRNA expression via its downstream transcription factors or via miRNA-target interactions with itself. To test this issue, we used APE1 siRNA to suppress the expression of the APE1 gene in human HOS cells and observed changes in the miRNA expression profile by miRNA microarray and qRT-PCR. We used a bioinformatics approach to analyze and predict the role of APE1 in regulating miRNAs expression. Pathway analysis showed that miRNAs and their target genes that were influenced by APE1 expression were involved in the alteration of pathways including developmental processes, regulation of cellular processes, cell signaling pathways, regulation of gene expression, and cancer pathways. Our study demonstrated that the expression of miRNA was directly affected by APE1 siRNA in osteosarcoma cells, and that these processes might also provide a novel pathway that APE1 uses to regulate gene expression through miRNAs and a possible functional role for the APE1 interactome.

\section{Materials and methods}

Cell culture. Human osteogenic sarcoma (HOS) cells were purchased from Cell Culture Center, Institute of Basic Medical Sciences, CAMS and PUMC, Beijing, China. HOS cells were maintained in DMEM, which was supplemented with $10 \%$ fetal bovine serum (HyClone), 100 units/ml penicillin and 100 $\mu \mathrm{g} / \mathrm{ml}$ streptomycin. Cells were cultured at $37^{\circ} \mathrm{C}$ in $5 \% \mathrm{CO}_{2}$.

APE1 knockdown. Recombinant adenovirus vector Ad5/ F35-APE1 siRNA and Ad5/F35-EGFP were constructed as described previously [10]. HOS cells were plated in six-well plates, transfected with Ad5/F35-APE1 siRNA or Ad5/F35EGFP at $20 \mathrm{MOI}$ for $4 \mathrm{~h}$ and then washed to remove the adenoviruses. After $48 \mathrm{~h}$ of culture, cells were harvested for further analysis.
Western blot. Western blot was used to confirm siRNAmediated down-regulation of APE1 protein in HOS cells. Total protein was prepared by standard procedures and quantified by the Bradford method. Protein extracts ( $10 \mu \mathrm{g}$ per sample) were run by $10 \%$ SDS-PAGE. After transferring and blocking, the membranes were incubated with mouse anti-APE1 $(1: 10,000$, SantaCruz) and $\beta$-actin monoclonal antibody (1:2,000, Sigma). After washing, membranes were then incubated with a horseradish peroxidase-conjugated secondary antibody (1:10,000, Pierce).

miRNA microarray assay. miRNA expression profiling was performed using Exiqon chip technology (Denmark). Ad5/F35-EGFP cells and Ad5/F35-APE1-siRNA cells were washed with pre-cooled PBS and total RNA was harvested using TRIzol (Invitrogen) and RNeasy Mini Kit (QIAGEN) according to the manufacturer's instructions. After having passed RNA measurement on the Nanodrop instrument, the samples were labeled using the miRCURY ${ }^{\mathrm{mm}} \mathrm{Hy} 3^{\mathrm{mm}} / \mathrm{Hy} 5^{\mathrm{mm}}$ Power labeling kit and hybridized on the miRCURY ${ }^{\mathrm{mm}}$ LNA Array (v.11.0). The samples were hybridized on a hybridization station following the scheme outlined in the sample submission. Scanning was performed with the Axon GenePix 4000B microarray scanner. GenePix pro V6.0 was used to read the raw intensity of the image. The intensity of green signal was calculated after background subtraction and the median value of four replicated spots of each probe on the same slide were calculated. We used the Median Normalization Method to obtain "Normalized Data"; Normalized Data $=$ (Foreground Background)/median, the median was the 50 percent quartile of miRNA intensity which was larger than 50 in all samples after background correction. The clustering was performed on the normalized data of miRNA. Only those miRNAs with Foreground-Back ground intensities larger than 50 in at least one sample have been included.

qRT-PCR. The SYBR Green PCR Master Mix (Applied Biosystems Foster City, CA) was used to confirm the candidate miRNAs obtained from the microarray data which changed more than 2 fold. MiRNA RT Primer and PCR primers were synthesized by Invitrogen. The template miRNA cDNA was constructed from total RNA by reverse transcriptase. The U6 gene was used for normalizing each sample.

Target genes prediction. miRNAs which had been confirmed by qRT-PCR were picked for target genes prediction. In order to enhance the reliability of predicted targets, we conducted integrated analyses of three databases including MicroCosm v5 (http://www.ebi.ac.uk/enright-srv/microcosm/ htdocs/targets/v5/), TargetScan 6.2 (http://www.targetscan. org/) and MICRORNA.ORG (http://www.microrna.org/ microrna/home.do).

Prediction of miRNA targeting APE1 3'UTR. MiRNA targets were predicted 4 different tools: miranda, pita, rnahybrid and targetscan, all of which were pre-computed and available within the miRecord database (http://mirecords.umn. edu/miRecords/). APE1-3'UTR sequence was retrieved from TargetScan 6.2 database (http://www.targetscan.org/). 

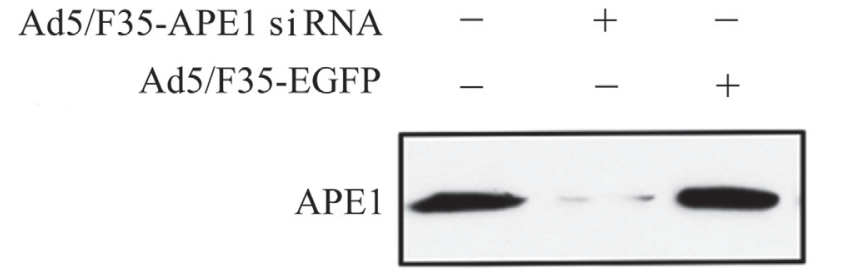

A

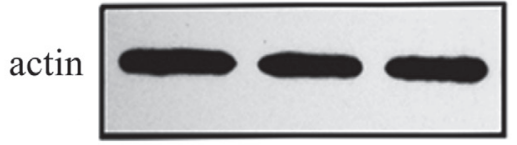

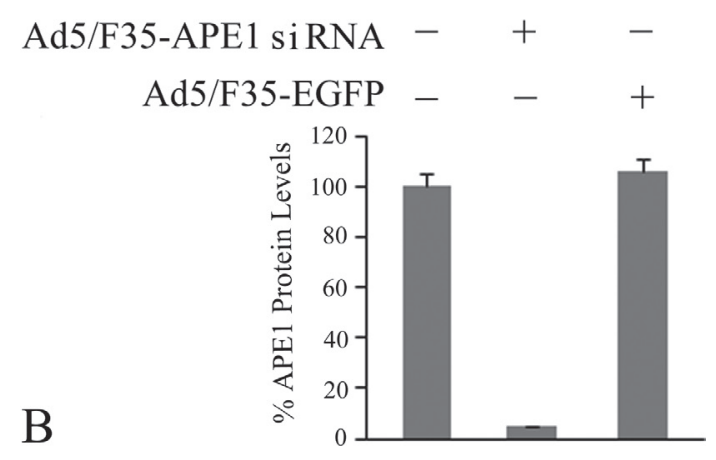

Figure 1. Knockdown of APE1 levels in HOS cells transfected with siRNA. A. Western blot. Samples were collected at 48 hours after APE1 siRNA treatment and Western blot was used to detect APE1 expression and reprobed with actin antibody as a loading control. B. Normalized APE1 levels after adjusting for loading.

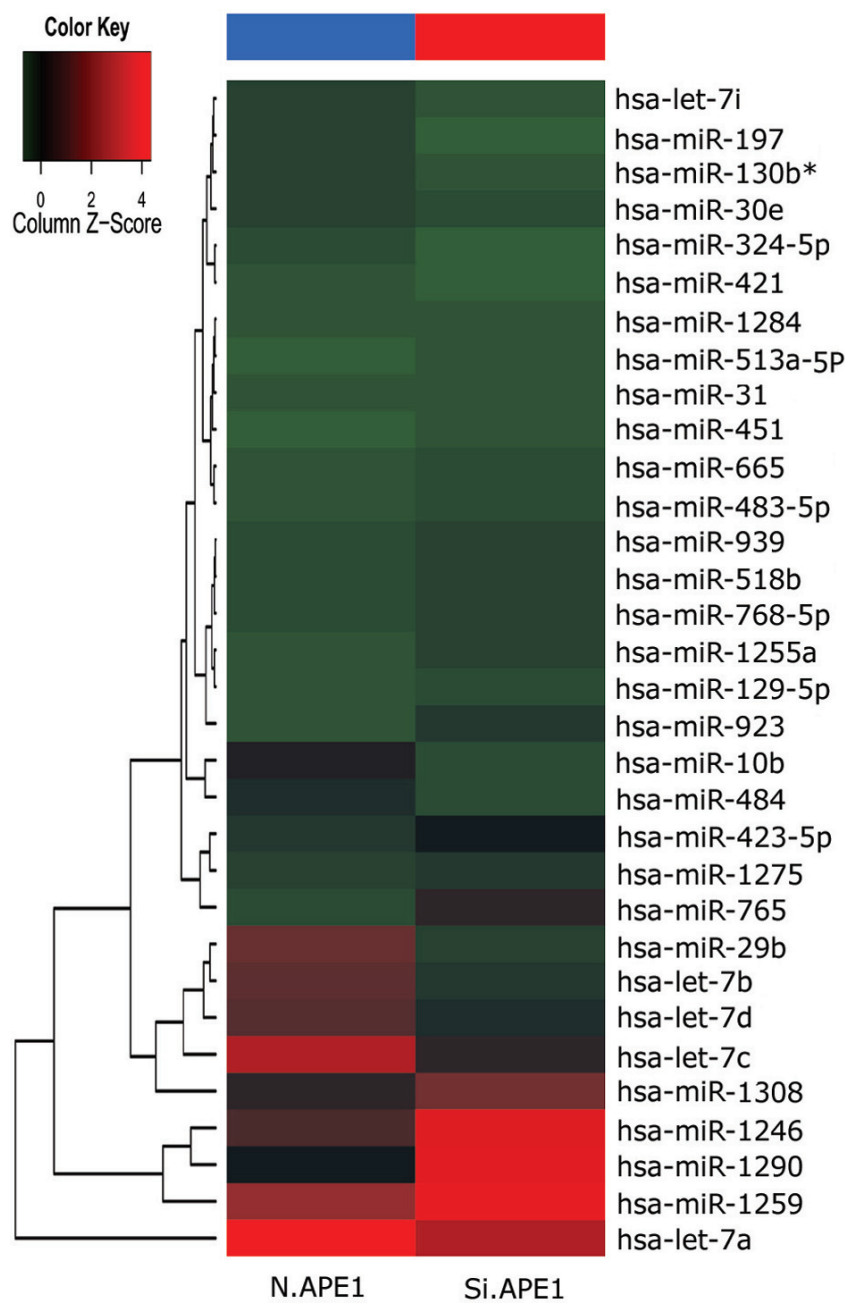

Figure 2. Hierarchical cluster analysis of miRNA expression. The heat map diagram shows the result of the two-way hierarchical clustering of genes and samples. Each row represents a miRNA and each column represents a sample. The miRNA clustering tree is shown on the left, and the sample clustering tree appears at the bottom. The color scale shown at the top illustrates the relative expression level of a miRNA.
Prediction of transcription factor putative binding sites in the miRNA promoters. Previous data have demonstrated that APE1 functions as a reduction-oxidation (redox) factor maintaining the DNA binding activity of transcription factors such as p53, NF- $\kappa B$, HIF-1a, HLF, PEBP2, ATF/CREB, Myb, Pax, Egr-1 and AP-1. The aberrant APE1 elevation was strongly correlated with the activation of these transcription factors. Therefore, we tried to identify the putative transcription factor binding regions in promoters of miRNAs.

Promoters (from 2500bp upstream to $500 \mathrm{bp}$ downstream of TSS, transcription start site) [20,21] of differentially expressed miRNAs were retrieved from UCSC genome browser(http://genome.ucsc.edu/). The binding sites of transcription factors HIF-1a and PEBP2 were predicted using EMBOSS (http://helixweb.nih.gov/emboss/html/fuzznuc. $\mathrm{html}$ ). The consensus sequences for HIF-1a and PEBP2 were [AG]CGTG and [AGT]ACC[AG]CA, respectively. For transcription factors other than HIF-1a or PEBP2, TRANSFAC 7.0 public database and the patser software (http://ural. wustl.edu/resources.html) were used for transcription factor binding site (TFBS) prediction. The cutoff of patser score was set to 0.9 .

Network analysis. The network is generated by integrating TFBS and miRNA target prediction results. The results were saved in data files describing links between transcription factors and miRNAs and then handled in cytoscape software (http://cytoscape.org).

Pathway analysis. The predicted miRNA targets found to be up- or down-regulated by APE1 were subjected to Kyoto Encyclopedia of Genes and Genomes (KEGG) PATHWAY Database (http://www.genome.jp/kegg/pathway.html) to uncover their association with molecular interaction and reaction networks.

Statistical analysis. Experimental results are expressed as mean values \pm standard error. Statistical analysis for microarray data and qRT-PCR was performed with Student's $t$ tests for comparison of two groups (SPSS software, version 13.0). Differences with $p<0.05$ were considered significant. 


\section{Results}

Confirmation of siRNA-mediated down-regulation of APE1 in HOS cells. The expression of APE1 protein after APE1 siRNA infection was examined by Western blot analysis. As shown in Fig.1, APE1 levels were decreased by 95\% after siRNA infection for $48 \mathrm{~h}$ as observed previously [10].

Down-regulating APE1 in HOS cells changes the miRNA expression profile. In this study, miRNA microarray was used to profile the changes of miRNA expression. The cluster analysis and a volcanl plot were applied to quickly identify the most-meaningful changes of miRNA and effectively target the most significant candidates for follow-up studies (Fig. 2 and Fig. 3). There were 32 miRNAs that changed $>2$-fold in the APE1 knocked-down HOS cells (Table 1). qRT-PCR was used to verify the results. For this approach, RNA was isolated from HOS cells treated with Ad5/F35-APE1 siRNA and Ad5/F35-EGFP in three independent experiments. The quantitative analysis demonstrated that 13 miRNAs showed statistically significant changes ( $>2$ fold) in expression levels following knock-down of APE1; 7 of them were up-regulated (hsa-miR-451, hsa-miR-1290, hsa-miR-765, hsa-miR-483-5p, hsa-miR-513a-5p, hsa-miR-129-5p and hsa-miR-31) and the other 6 miRNAs were down-regulated (hsa-miR-29b, hsa-miR197, hsa-let-7b, hsa-miR-324-5p, hsa-let-7i and hsa-miR-484) (Table 2).

Bioinformatics analysis of predicted target genes. To discover the effect of APE1 on miRNA expression profiles in osteosarcoma HOS cells, we predicted and analyzed the target genes of significant miRNAs by bioinformatics. In our

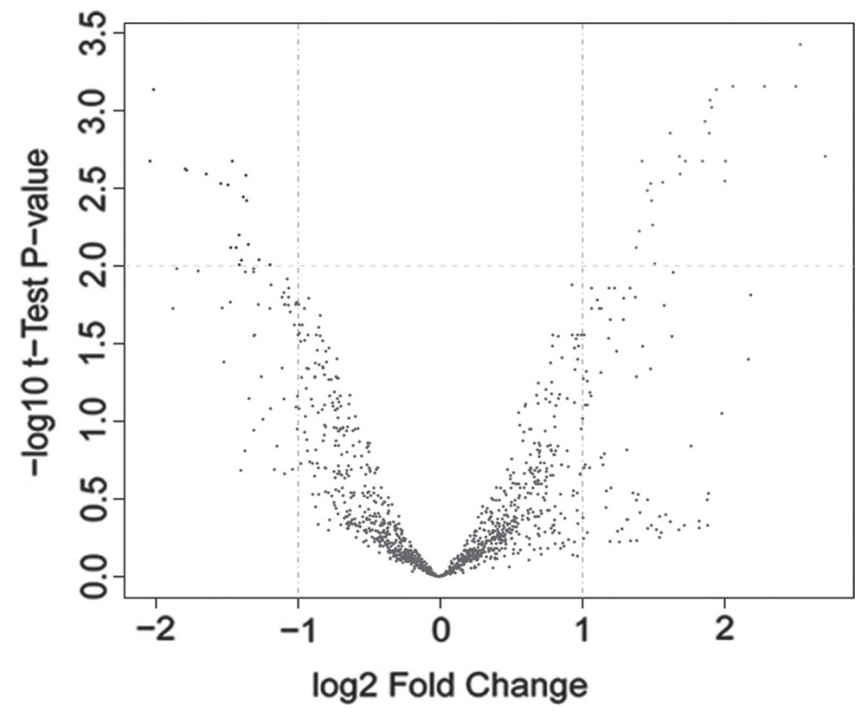

Figure 3. A volcano plot shows the significance miRNAs in APE1 knockdown HOS cells. The horizontal axis represents the fold change between normal HOS cells and APE1 knockdown HOS cells. The vertical axis represents the $p$-value of the $t$-test for the differences between samples $(p<0.01)$.

study, there were 3617 predicted target genes of up-regulated miRNAs and 1781 predicted targets of down-regulated miRNAs (Table 3).

Transcription factors binding sites. Generally, TFBS are 8-15 bp long and located in upstream regions near the TSS. We searched for potential TFBS in the flanking regions of

Table 1. miRNAs differentially expressed in APE1 siRNA transfected HOS cells and normal controls

\begin{tabular}{|c|c|c|c|c|c|}
\hline Up regulation of miRNAs & Fold $(>2)$ & p-value & Down regulation of miRNAs & Fold $(<0.5)$ & p-value \\
\hline hsa-miR-923 & 7.68 & 0.0000 & hsa-miR-29b & 0.29 & 0.0000 \\
\hline hsa-miR-451 & 7.21 & 0.0000 & hsa-miR-197 & 0.31 & 0.0000 \\
\hline hsa-miR-1290 & 5.50 & 0.0000 & hsa-let-7b & 0.36 & 0.0004 \\
\hline hsa-miR-765 & 5.19 & 0.0002 & hsa-miR-324-5p & 0.39 & 0.0011 \\
\hline hsa-miR-483-5p & 4.21 & 0.0000 & hsa-miR-421 & 0.43 & 0.0013 \\
\hline hsa-miR-513a-5p & 3.02 & 0.0005 & hsa-miR-10b & 0.44 & 0.0005 \\
\hline hsa-miR-1246 & 2.61 & 0.0012 & hsa-let-7i & 0.44 & 0.0000 \\
\hline hsa-miR-768-5p & 2.61 & 0.0006 & hsa-miR-130b* & 0.45 & 0.0021 \\
\hline hsa-miR-665 & 2.53 & 0.0000 & hsa-let-7c & 0.46 & 0.0000 \\
\hline hsa-miR-518b & 2.41 & 0.0026 & hsa-let-7a & 0.47 & 0.0000 \\
\hline hsa-miR-129-5p & 2.39 & 0.0019 & hsa-let-7d & 0.47 & 0.0001 \\
\hline hsa-miR-1255a & 2.35 & 0.0021 & hsa-miR-484 & 0.49 & 0.0006 \\
\hline hsa-miR-423-5p & 2.25 & 0.0000 & & & \\
\hline hsa-miR-1284 & 2.13 & 0.0000 & & & \\
\hline hsa-miR-939 & 2.08 & 0.0002 & & & \\
\hline hsa-miR-1275 & 2.06 & 0.0033 & & & \\
\hline hsa-miR-1259 & 2.05 & 0.0001 & & & \\
\hline hsa-miR-1308 & 2.01 & 0.0000 & & & \\
\hline hsa-miR-31 & 2.01 & 0.0002 & & & \\
\hline hsa-miR-30e & 2.00 & 0.0018 & & & \\
\hline
\end{tabular}




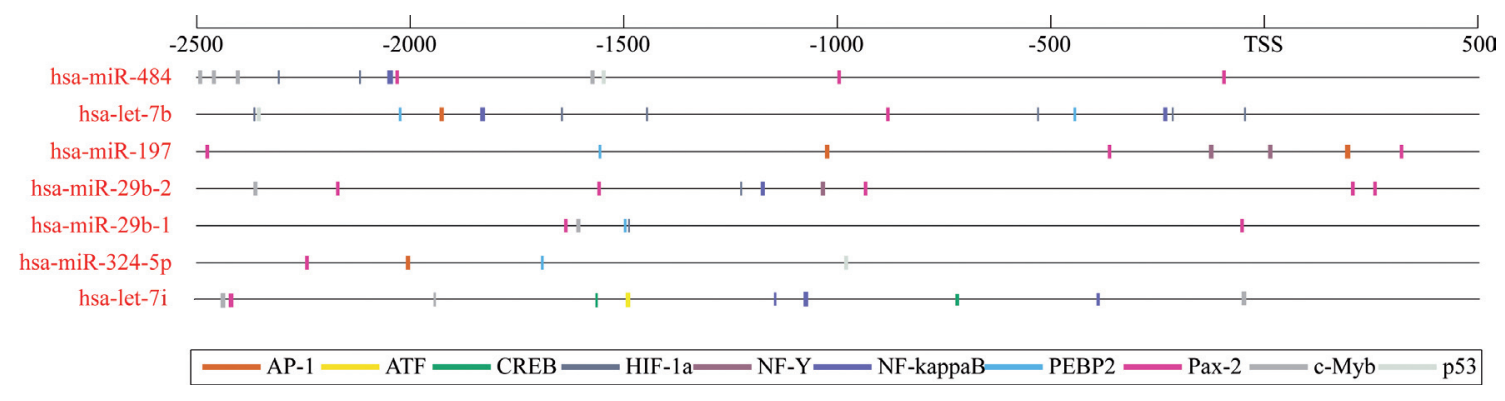

Figure 4. Putative transcription factors binding sites in down-regulated miRNAs promoters.

clustered and unclustered miRNAs to define their positional preferences and their class representation. TFBS can also be used to locate the core promoters of miRNA genes. In order to investigate the potential function of transcription factors mediated by APE1 in the regulation of miRNAs expression, the potential binding sites of miRNA promoters were analyzed using a bioinformatics approach. Because down-regulation of APE1 was strongly correlated with the decreased activation of transcription factors, down-regulated miRNAs after APE1 siRNA were chosen to identify the putative binding sites. Intriguingly, in six of the down-regulated miRNAs (Table 2), we found there are putative binding sites of NF- $\kappa B$, p53, HIF1a, AP-1, PEBP2, ATF, NF-Y, Pax-2,CREB and c-Myb in the promoters of several down regulated miRNAs (Fig 4 and Supplement Table 1).

MiRNA target to APE1-3'UTR. We then performed analysis of potential binding sequences for these ten miRNAs, miR-484, miR-513a-5p, miR-765, miR-324-5p, miR-451, miR483-5p, miR-197, miR-let-7i, miR-let-7b and miR-29b, in the 3'-UTR of APE1 mRNA and identified one putative binding site. site (the data not yet published). The „seed match“ se- quence of miR matched 4/9 nucleotides in positions 21-1112. The seed match sequence is important in guiding miRNA to specifically bind to the 3'complementary sequences in mRNA, and some upstream sequences may be also involved in binding. (Supplement Table 2)

Gene network analysis. We used the bioinformatics soft to generate a network from experimentally verified interactions of the biologically active, validated, down regulated miRNAs, transcription factors and APE1 (Supplement Figure 1). We further explored the regulation of the differentially regulated miRNAs by examining the interactions with the validated transcription factors at the predicted TFBS (Supplement Table 1), and found that 10 transcription factors interacted with the experimentally validated miRNAs (Supplement Figure 1). The interaction networks demonstrated a complex role for miRNAs in human osteosarcoma cells. Since miRNAs usually inhibit mRNA expression of their target genes, we expected that target mRNAs of the miRNAs down regulated in human osteosarcoma cells would be upregulated; several gene targets exhibited expression levels consistent with this expectation (shown in Table 4).

Table 2. Identification of differentially regulated miRNAs by qRT-PCR

\begin{tabular}{|c|c|c|c|}
\hline miRNAs & Sequence $5^{\prime} \rightarrow 3^{\prime}$ & qRT-PCR (APE1 siRNA/N) & $\mathrm{p}$ value \\
\hline \multicolumn{4}{|c|}{ Upregulated miRNAs } \\
\hline hsa-miR-451 & AAACCGTTACCATTACTGAGTT & 8.01 & 0.0000 \\
\hline hsa-miR-1290 & tGGAtttttGGAtCAGGGA & 4.62 & 0.0000 \\
\hline hsa-miR-765 & tGGAGGAGAAGGAAGGtGAtG & 6.28 & 0.0002 \\
\hline hsa-miR-483-5p & AAGACGGGAGGAAAGAAGGGAG & 5.54 & 0.0000 \\
\hline hsa-miR-513a-5p & ttCACAGGGAGGtGtCAt & 2.89 & 0.0001 \\
\hline hsa-miR-129-5p & CTTTTTGCGGTCTGGGCTTGC & 2.88 & 0.0019 \\
\hline hsa-miR-31 & AGGCAAGAtGCtGGCAtAGCt & 2.31 & 0.0000 \\
\hline \multicolumn{4}{|c|}{ Downregulated miRNAs } \\
\hline hsa-miR-29b & TAGCACCATTTGAAATCAGTGTT & 0.39 & 0.0000 \\
\hline hsa-miR-197 & ttCACCACCttCtCCACCCAGC & 0.44 & 0.0003 \\
\hline hsa-let-7b & tGAGGtAGtAGGttGtGtGGtt & 0.46 & 0.0005 \\
\hline hsa-miR-324-5p & CGCAtCCCCtAGGGCAttGGtGt & 0.48 & 0.0008 \\
\hline hsa-let-7i & TGAGGTAGTAGTTTGTGCTGTT & 0.49 & 0.0006 \\
\hline hsa-miR-484 & tCAGGCtCAGtCCCCtCCCGAt & 0.43 & 0.0000 \\
\hline
\end{tabular}


Supplement Table 1. Putative transcription factors binding sites in down-regulated miRNAs promoters

\begin{tabular}{|c|c|c|c|c|c|}
\hline Transcription factors & score & pval & hit_start & Down regulated miRNAs & hit_seq \\
\hline Pax-2 & 0.95238 & 0.000225 & $1614(+)$ & \multirow{13}{*}{ hsa-let-7b } & CCGAAACCC \\
\hline p53 & $9.64 \mathrm{E}-01$ & $1.14 \mathrm{E}-05$ & $143(-)$ & & AGACAAGTCT \\
\hline NF-kappaB & 0.90741 & $1.14 \mathrm{E}-05$ & $2262(-)$ & & GGGGCTCCCC \\
\hline NF-kappaB & $9.41 \mathrm{E}-01$ & $9.56 \mathrm{E}-07$ & $665(-)$ & & TGGGAAGTCCCT \\
\hline AP-1 & 0.90265 & $6.19 \mathrm{E}-05$ & $570(-)$ & & CCTCAGTCACC \\
\hline HIF-1a & 1 & 0 & $135(+)$ & & GCGTG \\
\hline HIF-1a & 1 & 0 & $854(+)$ & & ACGTG \\
\hline HIF-1a & 1 & 0 & $1053(+)$ & & GCGTG \\
\hline HIF-1a & 1 & 0 & $1967(+)$ & & GCGTG \\
\hline HIF-1a & 1 & 0 & $2282(+)$ & & ACGTG \\
\hline HIF-1a & 1 & 0 & $2451(+)$ & & ACGTG \\
\hline РEBP2 & 1 & 0 & $475(+)$ & & AACCACA \\
\hline PEBP2 & 1 & 0 & $2052(+)$ & & GACCGCA \\
\hline Pax-2 & 0.98214 & 3.04E-05 & $861(-)$ & \multirow{5}{*}{ hsa-miR-29b-1 } & GAGTTTGTG \\
\hline Pax-2 & 0.99405 & $1.51 \mathrm{E}-05$ & $2442(-)$ & & GGGTTTATT \\
\hline c-Myb & $9.38 \mathrm{E}-01$ & $6.84 \mathrm{E}-05$ & $890(+)$ & & GATAACTGGC \\
\hline HIF-1a & 1 & 0 & $1011(+)$ & & ACGTG \\
\hline PEBP2 & 1 & 0 & $1001(+)$ & & AACCACA \\
\hline Pax-2 & 0.95833 & 0.000145 & $328(-)$ & \multirow{7}{*}{ hsa-miR-29b-2 } & GTGTTTAAT \\
\hline Pax-2 & 0.95833 & 0.000145 & $939(+)$ & & CTTAAACAC \\
\hline Pax-2 & 0.95833 & 0.000145 & $1562(+)$ & & ATTAAACAC \\
\hline NF-Y & 0.9026 & 3.72E-05 & $1461(-)$ & & TTGATTGGTTG \\
\hline NF-kappaB & $9.33 \mathrm{E}-01$ & $9.54 \mathrm{E}-06$ & $1321(+)$ & & AGGAATTTCC \\
\hline $\mathrm{c}-\mathrm{Myb}$ & $9.38 \mathrm{E}-01$ & $6.84 \mathrm{E}-05$ & $135(+)$ & & ACTAACTGGC \\
\hline HIF-1a & 1 & 0 & $1273(+)$ & & ACGTG \\
\hline Pax-2 & 0.97619 & $6.07 \mathrm{E}-05$ & $256(-)$ & \multirow{4}{*}{ hsa-miR-324-5p } & GAGTTTCTT \\
\hline p53 & $9.16 \mathrm{E}-01$ & 7.05E-05 & $1516(-)$ & & GGACAAGTCC \\
\hline AP-1 & $9.20 \mathrm{E}-01$ & 2.67E-05 & $491(-)$ & & TTTTAGTCACT \\
\hline PEBP2 & 1 & 0 & $807(+)$ & & GACCACA \\
\hline Pax-2 & 0.95238 & 0.000225 & $2400(-)$ & \multirow{11}{*}{ hsa-miR-484 } & GAGTTTTTT \\
\hline Pax-2 & 0.96429 & 0.000126 & $467(-)$ & & GGGTTTATC \\
\hline Pax-2 & 0.96429 & 0.000126 & $1500(-)$ & & GAGTTTATA \\
\hline p53 & $9.13 \mathrm{E}-01$ & 8.19E-05 & $949(+)$ & & TGGCTTGTCT \\
\hline NF-kappaB & 0.94167 & $1.50 \mathrm{E}-06$ & $448(-)$ & & CGGGAAAGTCCCTA \\
\hline $\mathrm{c}-\mathrm{Myb}$ & $9.07 \mathrm{E}-01$ & 0.000218 & $6(-)$ & & TCCAGTTGAG \\
\hline c-Myb & $9.07 \mathrm{E}-01$ & 0.000218 & $38(+)$ & & GCCAACTGGG \\
\hline c-Myb & $9.07 \mathrm{E}-01$ & 0.000218 & $94(-)$ & & GGCAGTTATT \\
\hline c-Myb & $9.79 \mathrm{E}-01$ & 8.63E-06 & $923(-)$ & & GCCAGTTGGG \\
\hline HIF-1a & 1 & 0 & $192(+)$ & & ACGTG \\
\hline HIF-1a & 1 & 0 & $382(-)$ & & ACGTG \\
\hline Pax-2 & 0.96429 & 0.000126 & $23(-)$ & \multirow{5}{*}{ hsa-miR-197 } & GAGTTTCAG \\
\hline Pax-2 & 0.97024 & $7.26 \mathrm{E}-05$ & $2132(+)$ & & CTCAAACTC \\
\hline NF-Y & 0.9026 & $3.72 \mathrm{E}-05$ & $2369(+)$ & & GAACCAATAAG \\
\hline AP-1 & $9.12 \mathrm{E}-01$ & 3.83E-05 & $1471(+)$ & & TGTGACTCAAT \\
\hline PEBP2 & 1 & 0 & $942(+)$ & & AACCACA \\
\hline Pax-2 & 0.9881 & $2.28 \mathrm{E}-05$ & $2402(-)$ & \multirow{11}{*}{ has-let-7i } & GAGTTTAAT \\
\hline NF-kappaB & 0.91358 & $6.66 \mathrm{E}-06$ & $1188(+/-)$ & & GGGAATTCCC \\
\hline NF-kappaB & 1 & $9.56 \mathrm{E}-07$ & $371(+)$ & & GGGGATTCCC \\
\hline NF-kappaB & 0.91589 & $2.08 \mathrm{E}-05$ & $1150(+)$ & & GGGAAGTCCC \\
\hline NF-kappaB & 0.93333 & $2.13 \mathrm{E}-06$ & $1148(-)$ & & CCGGGAAGTCCCC \\
\hline $\mathrm{c}-\mathrm{Myb}$ & 0.90722 & 0.00021822 & $1949(-)$ & & GGAAGTTAAC \\
\hline c-Myb & 0.91753 & 0.00014923 & $2427(+)$ & & AATAACTGGC \\
\hline c-Myb & 0.91753 & 0.00014923 & $31(-)$ & & GCCAGTTCTG \\
\hline ATF & 0.91346 & 1.51E-05 & $1472(-)$ & & GGCAGGCGTCAGCG \\
\hline CREB & 0.90099 & 0.00015225 & $739(-)$ & & GTACGTCA \\
\hline CREB & 0.94262 & $1.35 \mathrm{E}-05$ & $1525(-)$ & & CCACGTCACCAG \\
\hline
\end{tabular}


Supplement Table 2. Putative differentially miRNAs binding sites in APE1

\begin{tabular}{|c|c|c|}
\hline miRNA & Position & Site \\
\hline \multirow{3}{*}{ hsa-miR-484 } & \multirow{3}{*}{ 21-27 of APE1 3'UTR } & 5’...CCCCUAAAUCACUUUGAGCCUGG... \\
\hline & & \multirow{2}{*}{$\begin{array}{c}|||||||| \\
3^{c} \quad \text { UAGCCCUCCCCUGACUCGGACU }\end{array}$} \\
\hline & & \\
\hline \multirow{3}{*}{ hsa-miR-513a-5p } & \multirow{3}{*}{ 129-135 of APE1 3'UTR } & $5^{\prime}$...UCCUCCAACCAGGCUCCUGUGAU... \\
\hline & & |||||| $\mid$ \\
\hline & & $3^{\prime} \quad$ UACUGUGGAGGGACACUU \\
\hline \multirow{3}{*}{ hsa-miR-765 } & \multirow{3}{*}{ 114-120 of APE1 3’UTR } & 5' ...GUAUAAAACUAGGAAUCCUCCAA... \\
\hline & & \multirow{2}{*}{ 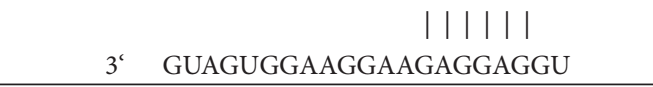 } \\
\hline & & \\
\hline \multirow{3}{*}{ hsa-miR-324-5p } & \multirow{3}{*}{ 1038-1042 of APE1 } & 5' ...AGCGCCAAGGCUUCGGGGAAUUACUGCA... \\
\hline & & ।।।। \\
\hline & & 3، UGUGGUUACGGGAUCCCCUACGC \\
\hline \multirow{3}{*}{ hsa-miR-451 } & \multirow{3}{*}{ 285-289 of APE1 } & 5’ ...CUUCGGUGGGUGACGCGGUA... \\
\hline & & |||| \\
\hline & & $3^{\prime} \quad$ UUGAGUCAUUACCAUUGCCAAA \\
\hline \multirow{3}{*}{ hsa-miR-483-5p } & \multirow{3}{*}{ 93-102 of APE1 } & \multirow{2}{*}{$\begin{array}{c}5^{5^{\prime}} \quad \text {...ACCCUUCUUUGUGCUCGG... } \\
\text { । । । । । । । }\end{array}$} \\
\hline & & \\
\hline & & $3^{\prime}$ GAGGGAAGAAAGGAGGGCAGAA \\
\hline \multirow{3}{*}{ hsa-miR-197 } & \multirow{3}{*}{ 340-345 of APE1 } & \multirow{2}{*}{ 5‘...AGCGUGGGAAAAAGGGAGCGGUGGCGGAAG... } \\
\hline & & \\
\hline & & $3^{\prime} \quad$ CGACCCACCUCUUCCACCACUU \\
\hline \multirow{3}{*}{ hsa-let-7i } & & $\begin{array}{ll}5^{\prime} & \text {...CGCGGUACAGCUGCCCAA... }\end{array}$ \\
\hline & 298-302 of APE1 & 1|| 1 \\
\hline & & $3^{\prime}$ UUGUCGUGUUUGAUGAUGGAGU \\
\hline & & $\begin{array}{ll}5^{c} & \text {...ACACACCCUAUGCCUACACCUUU... }\end{array}$ \\
\hline hsa-let-7b & $1107-1112$ of & |||| $\mid$ \\
\hline & & UUGGUGUGUUGGAUGAUGGAGU \\
\hline & & $\begin{array}{ll}5^{\prime} & \text {...GGCUGCCAUCGGGCCGGUGCA... }\end{array}$ \\
\hline hsa-miR-29b & $125-130$ of & | | | | \\
\hline & & $3^{c} \quad$ UUGUGACUAAAGUUUACCACGAU \\
\hline
\end{tabular}

Table 3. Validated and predicted target genes of differentially regulated miRNAs

\begin{tabular}{|c|c|c|c|}
\hline miRNAs & Location & Validated Target & Predicted Target \\
\hline hsa-miR-451 & chr 17: 27188387-27188458 & ABCB1, MIF,CAB39 & 20 \\
\hline hsa-miR-1290 & chr 1:19221565-19225642 & l & 133 \\
\hline hsa-miR-765 & chr 1:156905923-156906036 & NTRK3 & 364 \\
\hline hsa-miR-483-5p & chr 10:28246127-28246150 & I & 149 \\
\hline hsa-miR-513a-5p & chr X: 146294981-146295109 & $\mathrm{CD} 274$ & 904 \\
\hline hsa-miR-129-5p & chr 7:127845925-127849996 & NOTCH1, EIF2C3, CAMTA1 & 390 \\
\hline has-miR-31 & chr 9:21510114-21514184 & $\begin{array}{l}\text { RHOA, LATS2, PPP2R2A, CXCL12, KLF13, FIH, DMD, APRC5, MPRIP, } \\
\text { MMP16, FOXP3, HOXC13, TIAM1, JAZF1, NUMB, RET, NFAT5, FZD3, } \\
\text { RDX, YY1, ITGA5 }\end{array}$ & 368 \\
\hline hsa-miR-29b & chr 7:130560218-130564298 & $\begin{array}{l}\text { DNAJB11, SFPQ, CL1A, MCL1, CDK6, SP1, BACE1, DNMT3A, DNMT3B, } \\
\text { NID1, COL3A1, TET1, S100B, VEGFA, DNMT1, COL1A1, MMp15, } \\
\text { MMP24, FGG, COL4A1, ADAM12, GRN, MMP2, BCL2, FGA, FGB }\end{array}$ & 725 \\
\hline hsa-miR-197 & chr 1:110139515-110143589 & FUS1 & 217 \\
\hline hsa-let-7b & chr 22:46507566-46511648 & $\begin{array}{l}\text { LIN28B, CDK6, CCND1, CDC25A, RPIA, HMGA2, CCND2, PRDM1, } \\
\text { LIN28A, NRAS, CCNA2, ACTG1, CDC34, IFNB1, NR2E1, RDH10 }\end{array}$ & 577 \\
\hline hsa-miR-324-5p & chr 17:7124616-7128698 & GLI1, SMO & 141 \\
\hline hsa-let-7i & chr 12:62995466-62999549 & TLR4 & 24 \\
\hline hsa-miR-484 & chr 16:15735151-15739229 & l & 97 \\
\hline
\end{tabular}


Target genes pathway analysis. Pathway analysis by KEGG was used to uncover their association with molecular interaction and reaction networks. According to the results of data mining, we obtained lots of up-regulated pathways and down-regulated pathways $(\mathrm{p}<0.01)$ (Table 4$)$. Among all the differentially regulated pathways, several signaling pathways (e.g., TGF- $\beta$, Wnt, MAPK and p53) including cancer pathways appeared to be most affected. It suggested that APE1 might participate in these signaling pathways and the development of cancer by interaction with miRNAs. This represents novel evidence for the regulation by APE1 of signaling pathways via miRNAs.

\section{Discussion}

miRNAs are an abundant class of small non-protein-coding RNAs that function as negative gene regulators. With our growing understanding of biogenesis and biological functions of miRNAs, more evidence has indicated that they can repress the expression of important cancer-related genes and might prove useful in the diagnosis and treatment of cancer [22]. Although only a few reports have investigated the role of miRNA in osteosarcoma, previous studies using different osteosarcoma tissue samples and miRNA microarray assay identified several differentially expressed miRNAs, including miR-99b [23], miR-143 [24], miR-652 [25] that were identified to be down-regulated and related to metastasis, apoptosis, and tumorigenesis in osteosarcoma. In the osteosarcoma cell line U2OS, exogenous overexpression of miR-31 inhibited proliferation and induced apoptosis [26]. In addition, miR-99b and miR-31 were shown to target pathways with importance to tumor behaviors, including TGF- $\beta$, Wnt, MAPK, and the p53 pathways [23]. Hence, these studies suggest that miRNAs could be considered as novel osteosarcoma markers and potential therapeutic targets $[23,27]$. We found similar results in our microarray profile. miR-99b, miR-143 and miR-652 were down-regulated 1.89 fold, 1.61 fold, and 1.56 fold, respectively and miR-31 up-regulated more than 2 fold in HOS cells after APE1 knockdown. In contrast, we analyzed the global miRNA expression profile in APE1 knockdown human cervical cancer HeLa cells. The above miRNAs, except for miR-143, were down-regulated more than 2 fold in the APE1 knockdown HeLa cells when compared to wild type HeLa cells (unpublished data). These results suggest that miR-143 exhibits tissue-specific expression, while miR-99b, miR-31 and miR-652 were APE1 regulated miRNAs. We interpreted the association between the APE1 expression level and osteosarcoma-specific miRNAs to be due to a critical role that APE1 plays in osteosarcoma development and progression. Therefore, these results provide more evidence to validate new functions for APE1 in osteosarcoma.

Increasing evidence has indicated that miRNAs are involved deeply in gene regulation. The potential influence of miRNAs may impact almost every genetic pathway [28]. For investigating the interaction between APE1 and miRNAs, we

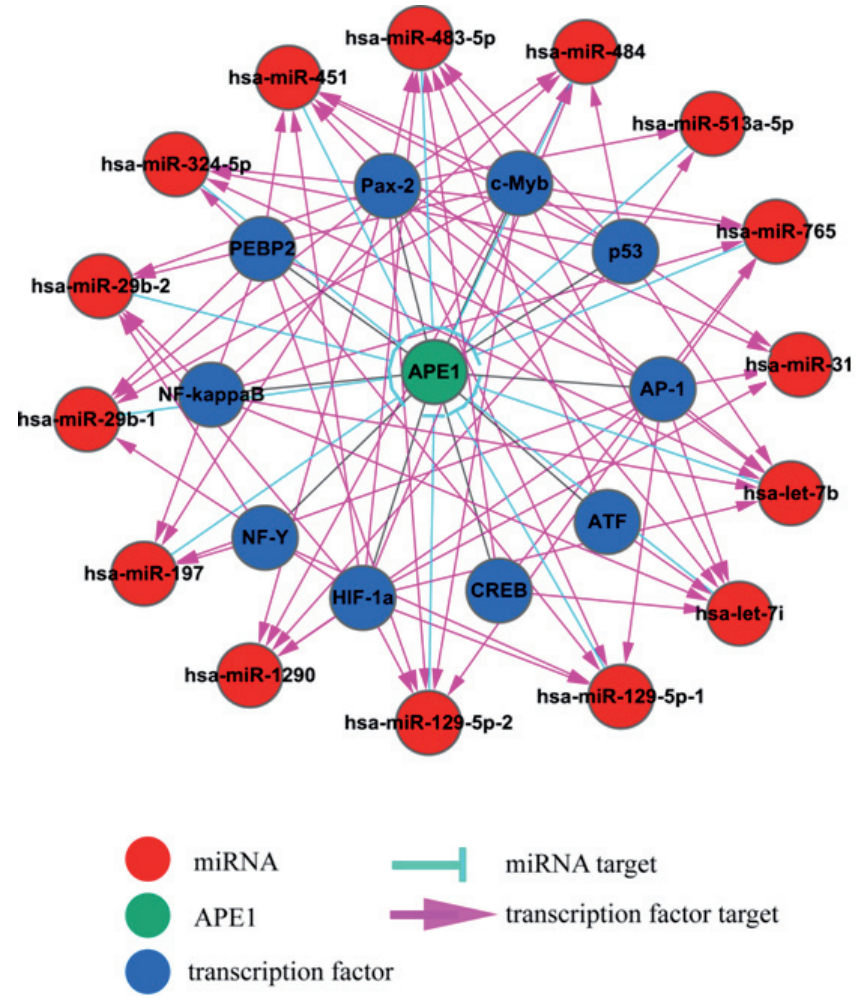

Supplement Figure 1 The network among APE1, transcription factors and differentially regulated miRNAs. Red circle, the differentially regulated miRNAs; Green circle, APE1 protein; Blue circle, the transcription factors regulated by APE1; Blue line, miRNAs were predicted to target APE1; Red line, the transcription factors have been indentified to regulate miRNAs; black line, transcription factors regulated by APE1

used a miRNA high-throughput screening assay to analyze the differentially expressed miRNAs in the presence and absence of APE1. As APE1 expression is ubiquitous in almost every human tissue and organ, the APE1 knock-down model of HOS cells by RNAi was designed for this approach. By controlling the expression of the APE1 gene, we observed 13 miRNA expression changes and we further confirmed these findings by quantitative real-time RT-PCR. In general, the up-regulated miRNAs may target genes to reduce their protein expression. In our study, among the seven up-regulated miRNAs, miR-451 was the most elevated, more than 7 fold in APE1 knock-down HOS cells. Previous studies have found that miR-451 can increase sensitivity of cells to doxorubicine in ovarian cancer [29] and breast cancer [30] by targeting the multidrug resistance gene MDR1. Another study reported by Chattopadhyay et al showed that acetylated APE1 enhanced Y-box-binding protein 1 (YB-1) expression, leading to the activation of MDR1 [31]. According APE1 has the miRNA cleaving properties [19], therefore, the result indicated that APE1 may directly or indirectly mediate miR-451 to establish a novel pathway in overcoming cell resistance, making it a potential target for the 
Table 4. Significant pathways analysis of miRNAs target genes (partial data)

\begin{tabular}{|c|c|c|c|}
\hline Pathway title & gene in pathway & p value & Symbol \\
\hline \multicolumn{4}{|c|}{ regulated by up-regulated miRNAs } \\
\hline TGF- $\beta$ signaling pathway & 24 & 0.0000 & $\begin{array}{l}\text { EP300, TNF, CREBBP, BMP2, CDKN2B, ID2, BMPR2, E2F5, SMAD6, BMPR1, } \\
\text { AGDF6, RPS6KB1, PPP2R1B, RHOA, SMAD7, TGFBR1, BMP4, ID4, TFDP1, CHRD, } \\
\text { THBS3, NOG, GDF5, PPP2R1A }\end{array}$ \\
\hline Adherens junction & 22 & 0.0000 & $\begin{array}{l}\text { EP300, FYN, CREBBP, CSNK2A2, WAS, TCF7, NLK, SRC, CTNND1, ACTN1, } \\
\text { PVRL4, PVRL1, PTPRB, WASF2, SSX2IP, TGFBR1, CTNNA2, WASF3, PVRL2, } \\
\text { IGF1R, RHOA FGFR1 }\end{array}$ \\
\hline Axon guidance & 30 & 0.0001 & $\begin{array}{l}\text { EFNB1, EPHA4, RGS3, EPHB3, EPHB1, SEMA3F, NRP1, EFNA5, NGEF, EPHA6, } \\
\text { SEMA4C, SRGAP1, PLXNB1,EFNA3, PAK6, EPHA8, EPHB2, ABLIM1, DPYSL2, } \\
\text { FYN, ABLIM2, GNAI2, RHOA, SRGAP2, DPYSL5, SEMA6A, SEMA4G, NFATC2, } \\
\text { FES, PAK7 }\end{array}$ \\
\hline Wnt signaling pathway & 31 & 0.0009 & $\begin{array}{l}\text { EP300, SFRP2, CREBBP, WNT5A, CTBP2, CSNK2A2, CER1, PPARD, CTNNBIP1, } \\
\text { NLK, PPP2R5B, NFATC2, VANGL1, CTBP1, TBL1XR1, PRICKLE2, PPP2R1B, } \\
\text { CAMK2D, WIF1, AMK2G, PPP2R1A, PLCB1, TBL1X, LRP6, AXIN2, TCF7, APC, } \\
\text { FRAT1, SIAH1, FZD5RHOA }\end{array}$ \\
\hline Long-term potentiation & 17 & 0.0020 & $\begin{array}{l}\text { EP300, ARAF, CREBBP, PPP1CB, GRIN2A, CALM2, CALM1, RPS6KA3, CACNA1C, } \\
\text { CAMK4, CAMK2D, CAMK2G, PLCB1, GRM1, RAP1B, GNAQ, CALM3 }\end{array}$ \\
\hline \multicolumn{4}{|c|}{ Regulated by down-regulated miRNAs } \\
\hline Small cell lung cancer & 19 & 0.0000 & $\begin{array}{l}\text { LAMA2, PTEN, CYCS, CCNE1, RARB, CDK6, PIAS4, BIRC2, LAMC1, COL4A2, } \\
\text { COL4A4, BCL2L1, COL4A1, LAMC2, TRAF4, IK3R1AKT3, COL4A6, E2F2 }\end{array}$ \\
\hline Pathways in cancer & 42 & 0.0000 & $\begin{array}{l}\text { MMP2, IGF1, LAMA2, PTEN, CYCS, FGF11, CCNE1, FAS, TPM3, ARNT, RARB, } \\
\text { NRAS, CDK6, PDGFRB, TGFBR1, CDC42, ACVR1C, EGFA, BIRC2, AMC1, } \\
\text { CASP3, PPARD, FASLG, WNT1, COL4A2, TGFB3, COL4A4, FZD5, FOS, TRAF4, } \\
\text { IGF1R, BCL2L1, E2F2, FZD4, COL4A6, COL4A1, ACVR1B, AMC2, PIAS4, IK3R1, } \\
\text { AKT3, DAPK1 }\end{array}$ \\
\hline TGF- $\beta$ signaling pathway & 14 & 0.0016 & $\begin{array}{l}\text { GDF6, ID1, ACVR2A, TGFBR1, ACVR1C, E2F5, TGFB3, SP1, IFNG, ZFYV, E16, } \\
\text { ACVR2B, ACVR1B, CHRD, SMAD6 }\end{array}$ \\
\hline p53 signaling pathway & 11 & 0.0054 & $\begin{array}{l}\text { IGF1, PTEN, CYCS, BBC3, CCNE1, FAS, STEAP3, CDK6, CASP3, CND2, } \\
\text { PPM1D }\end{array}$ \\
\hline Pancreatic cancer & 11 & 0.0074 & $\begin{array}{l}\text { CDK6, TGFBR1, CDC42, ACVR1C, VEGFA, TGFB3, BCL2L1, E2F2, ACVR1B, } \\
\text { PIK3R1, AKT3 }\end{array}$ \\
\hline
\end{tabular}

drug sensitization in osteosarcoma. Currently, further studies attempting to discover the mechanism of action on miR-451 and APE1 are underway in our laboratory.

In addition, based on previous studies which have demonstrated that down expression of APE1 was strongly correlated with the depressed activation of transcription factors [32], we presumed that the down-regulated miRNAs may be regulated by transcription factors via binding in promoters regions of miRNAs. The potential binding sites of miRNA promoters analyzed by bioinformatics suggested that APE1 may indirectly control the down-regulated let-7b, miR-324-5p, let-7i and $\mathrm{miR}-484$ via AP-1, NF- $\kappa$ B and c-Myb, respectively. Based on the bioinformatics tool, miR-29b which has been reported to be suppressed by NF- $\kappa \mathrm{B}$ and $\mathrm{c}-\mathrm{Myc}$ via promoter region binding [33] was not predicted in our study. Moreover we still need more evidence to confirm the possible effect of APE1 on expression of let-7b, let-7i, miR-324-5p and miR484 via transcription factors. Network showed there were also several genes known to be regulated by these miRNAs whose expression was decreased in human osteosarcoma cells (Supplement Figure 1), such as $\kappa \mathrm{B}, \mathrm{p} 53, \mathrm{c}-\mathrm{Myb}, \mathrm{HIF}-1 \alpha$ and
AP-1 et al.,which are involved in tumorigenesis and tumor progress [34].

In order to understand the function of miRNAs, bioinformatics analysis of the 13 miRNAs on their target genes was used as evidence to support the APE1-mediated regulation of miRNAs. KEGG pathway annotation was applied to the target gene pool. The results showed that important proliferative (MAPK, Wnt and ErbB), survival (p53, TGF- $\beta$ and mTOR), adhesive (adherens junction, focal adhesion and ECM-receptor interaction), oncogenic (prostate cancer, glioma, small cell lung cancer, colorectal cancer, melanoma and pancreatic cancer) and neural development (axon guidance, long-term potentiation and neurotrophin signaling pathway) signaling pathways were abundant among the significantly enhanced pathways. Some of them have already been reported to take part in an APE1-genes-network. For example, APE1 has been identified to take part in the p53 signaling pathway $[35,36]$ and in cancer development [10,37-40]. Small interfering RNA (siRNA) knockdown of endogenous APE1 impaired HMGB1mediated cytokine expression and MAPK activation in THP-1 cells [41]. Furthermore, noteworthy, Wang et al have reported 
the altered gene profile of HOS cells by gene array after knockdown of APE1 and verified that MAPK14 and TGF- $\beta 1$ were increased significantly [42]. Likewise, and interestingly, our study is the first to show that APE1 contributes to the Wnt and mTOR signaling pathways. These results not only support earlier findings, but also provide a new explanation of APE1related gene regulation pathways.

In conclusion, our data provide evidence that APE1 appears to have a direct influence on global miRNA expression, especially with regard to the tissue specific miRNAs in human osteosarcoma cells. It seems that APE1 may down regulate miRNAs via interaction with transcription factors. Certainly, there are several issues to be resolved in further studies. For instance, miRNAs which target APE1 should be identified and the binding sites of NF- $k$ B, p53, HIF-1 $\alpha$, AP-1, PEBP2, ATF, NF-Y, Pax-2, CREB and c-Myb in miRNA promoters also should be investigated. In addition, more experiments should be carried out to fully explain the relationship between APE1 and its potentially targeted pathways.

Acknowledgements: This work was supported by grant No. 81001000, No. 81172117 and No. 81101993 National Natural Science Foundation of China (NSFC).

\section{References}

[1] WANG LL. Biology of osteogenic sarcoma. Cancer J 2005; 11 : 294-305. http://dx.doi.org/10.1097/00130404-200507000$\underline{00005}$

[2] OTTAVIANI G AND JAFFE N. The epidemiology of osteosarcoma. Cancer Treat Res 2009; 152: 3-13. http://dx.doi. org/10.1007/978-1-4419-0284-9 1

[3] DELANEY TF, PARK L, GOLDBERG SI, HUG EB, LIEBSCH NJ, et al. Radiotherapy for local control of osteosarcoma. Int J Radiat Oncol Biol Phys 2005; 61: 492-498. http://dx.doi. org/10.1016/j.ijrobp.2004.05.051

[4] FERRARI S, BERTONI F, MERCURI M, PICCI P, GIACOMINI S, et al. Predictive factors of disease-free survival for non-metastatic osteosarcoma of the extremity: an analysis of 300 patients treated at the Rizzoli Institute. Ann Oncol 2001; 12: 1145-1150. http://dx.doi.org/10.1023/A:1011636912674

[5] TELL G, FANTINI D AND QUADRIFOGLIO F. Understanding different functions of mammalian AP endonuclease (APE1) as a promising tool for cancer treatment. Cell Mol Life Sci 2010; 67: 3589-3608. http://dx.doi.org/10.1007/s00018010-0486-4

[6] EVANS AR, LIMP-FOSTER M AND KELLEY MR. Going APE over ref-1. Mutat Res 2000; 461: 83-108. http://dx.doi. org/10.1016/S0921-8777(00)00046-X

[7] FISHEL ML AND KELLEY MR. The DNA base excision repair protein Ape1/Ref-1 as a therapeutic and chemopreventive target. Mol Aspects Med 2007; 28: 375-395. http://dx.doi. org/10.1016/j.mam.2007.04.005

[8] WANG D, LUO M AND KELLEY MR. Human apurinic endonuclease 1 (APE1) expression and prognostic significance in osteosarcoma: enhanced sensitivity of osteosarcoma to
DNA damaging agents using silencing RNA APE1 expression inhibition. Mol Cancer Ther 2004; 3: 679-686.

[9] WANG D, ZHONG ZY, LI MX AND LI ZP. Vector-based Ape1 small interfering RNA enhances the sensitivity of human osteosarcoma cells to endostatin in vivo. Cancer Sci 2007; 98: 1993-2001. http://dx.doi.org/10.1111/j.13497006.2007.00616.x

[10] XIANG DB, CHEN ZT, WANG D, LI MX, XIE JY, et al. Chimeric adenoviral vector Ad5/F35-mediated APE1 siRNA enhances sensitivity of human colorectal cancer cells to radiotherapy in vitro and in vivo. Cancer Gene Ther 2008; 15: 625-635. http://dx.doi.org/10.1038/cgt.2008.30

[11] BUSSO CS, LAKE MW AND IZUMI T. Posttranslational modification of mammalian AP endonuclease (APE1). Cell Mol Life Sci 2010; 67: 3609-3620. http://dx.doi.org/10.1007/ s00018-010-0487-3

[12] ESQUELA-KERSCHER A AND SLACK FJ. Oncomirs - microRNAs with a role in cancer. Nat Rev Cancer 2006; 6: 259-269. http://dx.doi.org/10.1038/nrc1840

[13] BARTEL DP. MicroRNAs: genomics, biogenesis, mechanism, and function. Cell 2004; 116: 281-297. http://dx.doi. org/10.1016/S0092-8674(04)00045-5

[14] CHO WC. OncomiRs: the discovery and progress of microRNAs in cancers. Mol Cancer 2007; 6: 60. http://dx.doi. org/10.1186/1476-4598-6-60

[15] ZHANG Y, GAO JS, TANG X, TUCKER LD, QUESENBERRY $\mathrm{P}$, et al. MicroRNA 125a and its regulation of the $\mathrm{p} 53$ tumor suppressor gene. FEBS Lett 2009; 583: 3725-3730. http:// dx.doi.org/10.1016/j.febslet.2009.10.002

[16] LE MT, TEH C, SHYH-CHANG N, XIE H, ZHOU B, et al.MicroRNA-125b is a novel negative regulator of p53. Genes Dev 2009; 23: 862-876. http://dx.doi.org/10.1101/ gad.1767609

[17] KUMAR M, LU Z, TAKWI AA, CHEN W, CALLANDER NS, et al. Negative regulation of the tumor suppressor p53 gene by microRNAs. Oncogene 2011; 30: 843-853. http://dx.doi. org/10.1038/onc.2010.457

[18] HE L, HE X, LOWE SW and HANNON GJ. microRNAs join the p53 network--another piece in the tumour-suppression puzzle. Nat Rev Cancer 2007; 7: 819-822. http://dx.doi. org/10.1038/nrc2232

[19] KIM WC, KING D AND LEE CH. RNA-cleaving properties of human apurinic/apyrimidinic endonuclease 1 (APE1). Int J Biochem Mol Biol 2010; 1: 12-25.

[20] XI Y, SHALGI R, FODSTAD O, PILPEL Y and JU J. Differentially regulated micro-RNAs and actively translated messenger RNA transcripts by tumor suppressor p53 in colon cancer. Clin Cancer Res 2006; 12: 2014-2024. http://dx.doi. org/10.1158/1078-0432.CCR-05-1853

[21] ZHOU X, REN Y, HAN L, MEI M, XU P, et al. Role of the AKT pathway in microRNA expression of human U251 glioblastoma cells. Int J Oncol 2010; 36: 665-672.

[22] BADER AG, BROWN D, STOUDEMIRE J and LAMMERS P. Developing therapeutic microRNAs for cancer. Gene Ther 2011; 18: 1121-1126. http://dx.doi.org/10.1038/gt.2011.79

[23] GOUGELET A, PISSALOUX D, BESSE A, PEREZ J, DUC A, et al. Micro-RNA profiles in osteosarcoma as a predictive 
tool for ifosfamide response. Int J Cancer 2011; 129: 680-690. http://dx.doi.org/10.1002/ijc.25715

[24] OSAKI M, TAKESHITA F, SUGIMOTO Y, KOSAKA N, YAMAMOTO Y, et al. MicroRNA-143 regulates human osteosarcoma metastasis by regulating matrix metalloprotease-13 expression. Mol Ther 2011; 19: 1123-1130. http://dx.doi. org/10.1038/mt.2011.53

[25] LULlA RR, COSTA FF, BISCHOF JM, CHOU PM, DE F BONALDO M, ET al. Identification of Differentially Expressed MicroRNAs in Osteosarcoma. Sarcoma 2011. 732690.

[26] CREIGHTON CJ, FOUNTAIN MD, YU Z, NAGARAJA AK, ZHU H, et al. Anderson, Molecular profiling uncovers a p53associated role for microRNA-31 in inhibiting the proliferation of serous ovarian carcinomas and other cancers. Cancer Res 2010; 70: 1906-1915. http://dx.doi.org/10.1158/0008-5472. CAN-09-3875

[27] MAIRE G, MARTIN JW, YOSHIMOTO M, CHILTONMACNEILL S, ZIELENSKA $M$ et al. Analysis of miRNA-gene expression-genomic profiles reveals complex mechanisms of microRNA deregulation in osteosarcoma. Cancer Genet 2011; 204: 138-146. http://dx.doi.org/10.1016/ j.cancergen.2010.12.012

[28] LEWIS BP, BURGE CB and BARTEL DP. Conserved seed pairing, often flanked by adenosines, indicates that thousands of human genes are microRNA targets. Cell 2005; 120: 15-20. http://dx.doi.org/10.1016/j.cell.2004.12.035

[29] VAN JAARSVELD MT, HELLEMAN J, BERNS EM and WIEMER EA. MicroRNAs in ovarian cancer biology and therapy resistance. Int J Biochem Cell Biol 2010; 42: 1282-1290. http://dx.doi.org/10.1016/j.biocel.2010.01.014

[30] KOVALCHUK O, FILKOWSKI J, MESERVY J, ILNYTSKYY Y, TRYNDYAK VP, et al. Involvement of microRNA-451 in resistance of the MCF-7 breast cancer cells to chemotherapeutic drug doxorubicin. Mol Cancer Ther 2008; 7: 2152-2159. http://dx.doi.org/10.1158/1535-7163.MCT-08-0021

[31] CHATTOPADHYAY R, DAS S, MAITI AK, BOLDOGH I, XIE J, et al. Regulatory role of human AP-endonuclease (APE1/Ref-1) in YB-1-mediated activation of the multidrug resistance gene MDR1. Mol Cell Biol 2008; 28: 7066-7080. http://dx.doi.org/10.1128/MCB.00244-08

[32] BHAKAT KK, MANTHA AK and MITRA S. Transcriptional regulatory functions of mammalian AP-endonuclease (APE1/Ref-1), an essential multifunctional protein. Antioxid Redox Signal 2009; 11: 621-638. http://dx.doi.org/10.1089/ ars.2008.2198
[33] MOTT JL, KURITA S, CAZANAVE SC, BRONK S, WERNEBURG NW et al. Transcriptional suppression of mir-29b-1/mir-29a promoter by c-Myc, hedgehog, and NFkappaB. J Cell Biochem 2010; 110: 1155-1164. http://dx.doi. org/10.1002/jcb.22630

[34. SARA M WEIS and DAVID A CHERESH. Tumor angiogenesis: molecular pathways and therapeutic targets.Nature Medicine 2011; 17: 1359-1370. http://dx.doi.org/10.1038/ nm.2537

[35] HAFSI $\mathrm{H}$ and HAINAUT P. Redox Control and Interplay Between p53 Isoforms: Roles in the Regulation of Basal p53 Levels, Cell Fate, and Senescence. Antioxid Redox Signal 2011; 15 : 1655-1667. http://dx.doi.org/10.1089/ars.2010.3771

[36] BUSSO CS, IWAKUMA T AND IZUMI T. Ubiquitination of mammalian AP endonuclease (APE1) regulated by the p53MDM2 signaling pathway. Oncogene 2009; 28: 1616-1625. http://dx.doi.org/10.1038/onc.2009.5

[37] ZHANG Y, WANG J, XIANG D, WANG D and XIN X. Alterations in the expression of the apurinic/apyrimidinic endonuclease-1/redox factor-1 (APE1/Ref-1) in human ovarian cancer and indentification of the therapeutic potential of APE1/Ref-1 inhibitor. Int J Oncol 2009; 35: 1069-1079.

[38] KELLEY MR, CHENG L, FOSTER R, TRITT R, JIANG J, et al. Elevated and altered expression of the multifunctional DNA base excision repair and redox enzyme Ape1/ref-1 in prostate cancer. Clin Cancer Res 2001; 7: 824-830.

[39] ROBERTSON KA, BULLOCK HA, XU Y, TRITT R, ZIMMERMAN E, et al: Altered expression of Ape1/ref-1 in germ cell tumors and overexpression in NT2 cells confers resistance to bleomycin and radiation. Cancer Res 2001; 61: 2220-2225.

[40] WANG D, XIANG DB, YANG XQ, CHEN LS, LI MX, et al. APE1 overexpression is associated with cisplatin resistance in non-small cell lung cancer and targeted inhibition of APE1 enhances the activity of cisplatin in A549 cells. Lung Cancer 2009; 66: 298-304. http://dx.doi.org/10.1016/ j.lungcan.2009.02.019

[41] YUK JM, YANG CS, SHIN DM, KIM KK, LEE SK, et al. A dual regulatory role of apurinic/apyrimidinic endonuclease 1/redox factor-1 in HMGB1-induced inflammatory responses. Antioxid Redox Signal 2009; 11: 575-588. http://dx.doi. org/10.1089/ars.2008.2196

[42] WANG D, ZHONG ZY, LI ZP, YANG ZZ AND KELLEY MR. The altered gene profile of human osteosarcoma cell after knock-down of double functional gene apurinic/apyrimidinic endonuclease. ACTA Academiae Medicinae Militaris Tertiae 2007; 29: 1-4. 